\title{
Quaderni
}

QUADERNI Communication, technologies, pouvoir

81 | Printemps 2013

L'humain médicament

\section{Les thérapies innovantes au prisme de l'évaluation des politiques publiques et de l'appréhension du risque}

Advanced therapies through public policies and risk assessment

\section{Nathalie Schiffino}

\section{(2) OpenEdition}

Journals

Édition électronique

URL : http://journals.openedition.org/quaderni/710

DOI : $10.4000 /$ quaderni. 710

ISSN : 2105-2956

Éditeur

Les éditions de la Maison des sciences de l'Homme

Édition imprimée

Date de publication : 5 juin 2013

Pagination : 45-60

Référence électronique

Nathalie Schiffino, "Les thérapies innovantes au prisme de l'évaluation des politiques publiques et de l'appréhension du risque », Quaderni [En ligne], 81 | Printemps 2013, mis en ligne le 05 juin 2015, consulté le 30 avril 2019. URL : http://journals.openedition.org/quaderni/710 ; DOI : 10.4000/ quaderni.710 


\section{$D$ ossier}

Les thérapies innovantes au prisme de l'évaluation des politiques publiques et de l'appréhension du risque

\section{Nathalie Schiffino}

Professeur de science politique, UCL
Investiguer la régulation publique des thérapies innovantes (thérapie génique, cellulaire et ingénierie tissulaire dont l'introduction de ce dossier fournit notamment une présentation juridique) est passionnant car il s'agit d'analyser les décisions publiques adoptées quant à des procédés médicaux qui créent du neuf dans notre société contemporaine. Les thérapies innovantes (TI) relèvent le défi d'employer, comme médicaments, des composants du vivant (des gènes, des cellules et notamment des cellules souches, des tissus comme la peau). La recherche et développement (R\&D), les essais cliniques et la commercialisation relatifs à de tels produits thérapeutiques introduisent de facto du nouveau dans la régulation publique. Comment les décideurs politiques doivent-ils accompagner le développement et les applications des connaissances scientifiques et médicales ? Comment doivent-ils les évaluer ? Tirant des leçons du passé (controverses autour de l'amiante, du tabac, des OGM, etc.), doivent-ils axer l'évaluation sur des risques définis principalement sous l'angle sanitaire et par une expertise mobilisée pour l'examen au cas par cas des dossiers ? Peuvent-ils prendre en compte l'impact sociétal des TI, c'est-à-dire apprécier leur potentiel économique, identifier les sources possibles de controverses, repérer les interlocuteurs dans la société civile, estimer les changements potentiels pour les soins de santé et la politique hospitalière, etc. ?

Si la règlementation européenne est en avance par rapport à celles des États-Unis et du Japon, ainsi que par rapport aux produits qu'elle encadre ${ }^{1}$, une innovation en termes d'évaluation de politique publique consisterait à anticiper d'emblée l'impact sociétal des TI, tel qu'entendu ci-dessus. 
Cette optique n'est pas complètement absente du dispositif actuel de régulation. Par exemple, de manière générale, le Comité économique et social européen (CESE), dans son avis de 2011, appelle le groupe d'experts "de haut niveau chargé d'examiner les domaines d'action énumérés» à «la prévoyance, l'ambition et une approche globale $»^{2}$. Une telle approche, alors que nous sommes encore relativement au début du processus de régulation, pourrait englober l'ensemble des étapes du cycle d'une politique publique (mise à l'agenda, programme d'action publique, sa mise en œuvre et son évaluation), mais aussi et surtout les interactions complexes entre les acteurs de mise en œuvre (les décideurs publics aux différents échelons de pouvoir : européen, national, local), les groupes cibles (les firmes pharmaceutiques, petites et moyennes entreprises, les centres de recherche) et les bénéficiaires finaux (les patients) de la politique.

La littérature scientifique sur la régulation des biotechnologies rouges et vertes depuis les années 1990 (notamment les travaux de Gilbert, Lascoumes, Joly) ${ }^{3}$ a démontré que la rationalité de cette régulation n'était pas uniquement technique et rationnelle; elle supposait une conception globale du risque, et devait inclure des formes participatives. C'est précisément le défi que les TI amènent dans le champ des connaissances en évaluation des politiques publiques. Par ailleurs, au-delà de la démarche classique en évaluation (exposée entre autres par Rossi, Monnier, Perret) ${ }^{4}$, les TI posent la question des leçons que nous avons tirées des crises sanitaires passées pour évaluer différemment, de façon plus globale, l'impact des objets innovants et porteurs de risques.
À partir de la littérature sur le risque et l'évaluation des politiques publiques, notre contribution formule l'hypothèse que la régulation publique des TI reste principalement ancrée dans une conception traditionnelle de l'évaluation, centrée sur le rôle des agences administratives, le poids de l'expertise scientifique et une prépondérance des interactions avec les groupes cibles (c'està-dire les firmes pharmaceutiques, petites et moyennes entreprises, les centres de recherche) plutôt qu'avec les bénéficiaires finaux (c'est-àdire les patients et, dans une optique plus large, les citoyens).

Afin d'étayer cette hypothèse, trois axes problématiques sont développés. Tout d'abord, nous définissons en quoi consiste une évaluation de politique publique et nous observons ce qui est prévu par la régulation publique dans le cas des TI. Ceci nous permet de constater que l'évaluation est entendue comme un examen scientifique au cas par cas, avec un questionnement éthique ad hoc. Deuxièmement, nous interrogeons l'appréhension du risque (sanitaire et éthique) aujourd'hui et nous remarquons sa prise en charge par des agences administratives, y compris en ce qui concerne les TI. Ceci nous conduit à mettre en avant la logique de décentrement de la régulation publique du niveau européen vers des agences administratives nationales activant des comités d'experts spécialisés. Enfin, nous rendons compte des modalités d'interaction prévues avec les groupes cibles et les bénéficiaires finaux pour élargir la dynamique de régulation. Ce qui nous amène à cerner la place limitée conférée aux bénéficiaires finaux dans le processus.

Potentiellement, cette triangulation entre les 
thèmes de l'évaluation, du risque et de la démocratie peut conduire au constat que peu de leçons sont mises en œuvre pour changer la logique de régulation publique axée sur l'expertise et la limitation de l'intervention du public dans des secteurs scientifiques et médicaux de pointe. Toutefois, nous nuançons une conclusion qui serait alors trop rapide. En effet, la prise en compte d'arguments éthiques, l'ouverture des procédures administratives à une consultation du public, et la mobilisation des patients attestent d'une adaptation de l'évaluation, et plus largement de la régulation publique, à une dimension sociétale.

Notre propos s'appliquera principalement à l'échelon de l'Union européenne (UE) et concernant la Belgique. Le premier médicament issu de la thérapie cellulaire et disposant d'une autorisation européenne de mise (AMM) sur le marché depuis 2009 est belge. La Belgique participe à la R\&D et à l'essor économique du secteur par des spin-off largement issues des universités ou des petites et moyennes entreprises. Les pouvoirs publics encadrent des associations professionnelles du secteur à l'échelon fédéral (association belge de l'industrie biotechnologique : Bio.be) et régional (BioWin en Wallonie et FlandersBio en Flandre). Outre l'application du règlement européen (CE) n¹394/2007 et la transposition de la directive européenne 2004/23/CE par la loi fédérale du 19 décembre 2008, les pouvoirs publics belges se sont intéressés à la problématique et une agence administrative, créée en 2007 pour réguler les médicaments et les produits de santé, intervient dans le domaine des $\mathrm{TI}^{5}$.

Chaque section de notre contribution reposera principalement sur la littérature scientifique en analyse de politique publique. Basée sur une méthode empiriste, elle articulera des éléments opérationnels liés à l'appareil politico-administratif, aux décisions publiques adoptées et aux interactions entre les acteurs de mise en œuvre (les décideurs publics), les groupes cibles (les firmes pharmaceutiques, entreprises et centres de recherche) et les bénéficiaires finaux (les patients et plus largement les citoyens).

\section{Évaluation de la politique ou expertise sur l'objet régulé ?}

En théorie, une évaluation de politique consiste à " apprécier les effets bénéfiques ou néfastes, attendus ou imprévus, directs ou secondaires, d'une action publique et à en informer les décideurs politiques, les fonctionnaires et les citoyens. Elle présente certes un instrument de contrôle, mais aussi, et surtout à notre avis, une aide à la décision, un outil de gestion et un catalyseur pour l'amorce de processus d'apprentissage collectif au sein de la fonction publique $»^{6}$. La potentialité que comporte l'évaluation de tirer des leçons d'une action publique peut d'ailleurs acquérir une dimension sociétale si ces leçons sont diffusées auprès des groupes cibles et des bénéficiaires finaux et/ou donnent lieu à des ajustements qui les concernent. Si une évaluation qui porte sur l'ensemble d'une politique publique n'est pas instrumentalisée, elle peut être un activateur de démocratie. En analysant la mise à l'agenda, la mise en œuvre et, le cas échéant, les déficits de mise en œuvre d'une politique publique, l'évaluation peut ouvrir un débat sur l'action publique et l'objet qu'elle entend réguler. Sur un objet scientifique, médical, technique comme les TI, 
l'évaluation ouvrirait une fenêtre d'opportunité pour réfléchir à l'articulation entre science, société et éthique ${ }^{7}$. C'est à cela que renvoie une évaluation à dimension sociétale.

Certes, l'évaluation de politique publique peut être définie de façon plus stricte par les outils qu'elle emploie, tels des analyses d'impact, fréquemment utilisées en santé publique. Pour apprécier les effets bénéfiques ou néfastes de produits, l'évaluation recourt à des (quasi-) expérimentations, avec des mesures de variables : « the critical issue in impact evaluation is whether a program produces desired effects over and above what would have occurred without the intervention or, in some cases, with an alternative intervention $»^{8}$. Dans le cas des TI, les essais cliniques ayant été développés suite à la $\mathrm{R} \& \mathrm{D}$, cela supposerait de comparer les apports des TI à un groupe de patients, par rapport à un groupe-contrôle, et d'en inférer la plus-value d'une politique publique régulant les $\mathrm{TI}^{9}$.

Les textes juridiques portent davantage sur une évaluation scientifique de dossiers, au cas par cas, par une expertise spécialisée dans le secteur. Certes, le règlement (CE) nº1394/2007 est replacé dans un cadre plus large lorsque, dans son « considérant $" \mathrm{n}^{\circ} 8$, il énonce qu'il respecte : «les principes inscrits dans la Charte des droits fondamentaux de l'UE (...), les droits de l'homme et la dignité de l'être humain à l'égard des applications de la biologie et de la médecine ». Mais le «considérant » $n^{\circ} 9$ montre dans la foulée que l'évaluation est conçue au sens strict, comme "une évaluation scientifique unique de la qualité, de la sécurité et de l'efficacité du produit ». Il s'agit bien d'une évaluation de l'objet (les TI), et non de l'ensemble de la politique publique l'encadrant, à ses différentes étapes. L'article 25 du règlement $n^{\circ} 1394 / 2007$ atteste que le réexamen des mesures prévues, que l'on pourrait comparer à une évaluation, se situe dans un périmètre limité : il porte sur les types de TI approuvées, sur l'impact du progrès technique sur le règlement lui-même, pour en redéfinir le champ d'application, mais il ne mentionne pas l'impact du règlement sur les bénéficiaires finaux ou les groupes cibles de la politique.

L'évaluation d'une politique publique repose sur quatre critères : son effectivité, son efficacité, son efficience, et sa pertinence ${ }^{10}$. L'effectivité est directement liée à l'analyse d'impacts : elle mesure le degré d'adéquation entre les objectifs assignés à une politique publique et le changement constaté sur le comportement des groupes cibles s'adaptant à la mise en œuvre de la politique publique. L'efficacité évalue l'adéquation entre les objectifs et l'amélioration de la situation des bénéficiaires finaux grâce à la mise en œuvre de la politique publique. Si les résultats ainsi escomptés sont atteints, une analyse de l'efficience de la politique permet d'évaluer le rapport entre les ressources investies dans la politique (son coût) et les effets obtenus (bénéfices). Enfin, la pertinence interroge les liens entre les objectifs assignés à une politique publique et la pression du problème auquel elle prétend répondre (ce qui inclut une dimension politique évidente).

De plus, il est utile de comparer les principes de base en éthique biomédicale, rappelés dans les avis du Comité consultatif de Bioéthique de Belgique (CCB) abordant les $\mathrm{TI}^{11}$, avec les critères d'efficience et d'efficacité d'une politique 
publique. Le CCB et les agences administratives font référence à la « balance bénéfice/risque » que l'on retrouve dans l'estimation de l'efficience d'une politique. Ainsi, dans son avis $n^{\circ} 52$, le $\mathrm{CCB}$ rappelle que le principe de base en éthique biomédicale appelé " principe de bienfaisance » consiste à «maximiser la quantité de «bien ». Appliqué au contexte médical, cela implique que les frais exposés doivent être rentabilisés. Effectuer des essais inutiles ou dont les résultats ne sont pas probants, est un gaspillage de ressources rares. Davantage de "bien " peut être créé par cet argent sur la base de moyens judicieusement affectés $»^{12}$. Dans la même perspective, l'Agence nationale (française) de sécurité du médicament et des produits de santé (ANSM) explique que l'objectif poursuivi par le règlement (CE) $n^{\circ} 1394 / 2007$ est de «mettre en place les conditions nécessaires au recueil d'information en continu et dans la pratique réelle, permettant de s'assurer que le produit présente bien une ba-lance bénéfice/risque favorable $»^{13}$.

Le caractère éthique de l'évaluation pourrait élargir le périmètre de celle-ci, depuis le champ scientifique sur l'objet sensu stricto, vers la dimension sociétale de l'impact de la politique publique pour tous les acteurs concernés. L'intégration de la dimension éthique dans l'évaluation des TI laisse à penser que les décideurs publics ont tiré des leçons du recours à l'évaluation éthique réalisée en biomédecine et de l'intégration d'évaluation anthropologique et socio-économique dans le secteur des OGM agro-alimentaires. C'est le cas au niveau national mais aussi européen. Ainsi, l'article 21 du règlement européen (CE) $n^{\circ} 1394 / 2007$ relatif à la composition du Comité des thérapies innovantes (CAT) prévoit que le comité comprenne des experts en matière de gestion du risque et d'éthique.

Néanmoins, il s'agit d'une expertise au cas par cas, sur base d'auto-saisine par le CCB ou de questions adressées par les mandataires politiques, mais qui sont ad hoc, non planifiées, non organisées. La formulation des questions dont se saisit le Comité fournit aussi un indice important de son intervention et de la portée de cette dernière. Le CCB précise dans ses avis les éléments auxquels il répond et ceux qu'il exclut de sa réponse. À titre d'exemple, l'avis $n^{\circ} 45$ sur les banques de matériels corporels humains comporte plusieurs expressions : «le présent avis ne concerne que (...). Il ne traite pas des (...). De même, sont exclues du présent avis (...). Sont également exclues du présent avis (...) $\gg^{14}$. Cette précision procède d'une logique scientifique d'expertise pointue à laquelle on ne «peut » pas demander l'évaluation sociétale d'un objet.

Or, la façon dont les questions sont posées, dont les problèmes publics sont formulés, influence le type de réponses qui seront apportées au problème public de départ ${ }^{15}$. Gardella souligne fort à propos que la culture publique qui prévaut pour le traitement et la résolution des problèmes publics n’est « pas un système de représentations abstraites ou inconscientes mais bien l'expression d'un ensemble de fonctions sociales ou de tensions » dans le système social. D'où l'importance placée ici sur une évaluation sociétale des TI. Des acteurs de mise en œuvre, groupes cibles et bénéficiaires finaux mènent « des activités stratégiques et symboliques permettant d'imposer » ce que Muller appelle un référentiel ${ }^{16}$. Les acteurs développent notamment une «matrice de procé- 
dés dramatiques et rhétoriques qui configurent le sens d'un problème $»^{17}$.

\section{Nouvelle appréhension du risque ou prévalence des agences et de l'expertise ?}

Une telle conception de l'évaluation dans une politique du risque est liée à la façon dont ce dernier est perçu et construit en problème public, étant entendu qu'une politique publique est par ailleurs supposée y apporter une solution (en théorie efficace, efficiente, effective et pertinente). Le risque est généralement défini comme la probabilité d'un évènement futur, positif ou négatif ${ }^{18}$. Et lorsque les conséquences d'un évènement sont négatives, le risque est défini comme la probabilité qu'un danger survienne ${ }^{19}$. Dans une société où serait nourrie l'utopie d'une sécurité et d'une santé parfaite ${ }^{20}$, d'aucuns évoqueraient la possibilité d'une "société du risque zéro ». Pourtant, dans des situations de risque potentiel comme celles présentées par les TI, la traditionnelle gestion des risques, reposant sur la théorie des choix rationnels et le Technology Assessment, n'est d'aucun secours ${ }^{21}$. Notre société contemporaine du risque, pour reprendre le terme du sociologue allemand Beck, vit fondamentalement une tension liée à la façon dont nous concevons et prenons en charge le risque lorsqu'une innovation (en l'occurrence médicale) promet des « bienfaits » (pour reprendre les termes évaluatifs du principe éthique susmentionné) tout en comportant des incertitudes (liées au manque de connaissances sur les effets de l'objet).

Or, selon Beck, le passage d'une société industrielle ou moderne, où le problème central était la répartition des richesses, à une société post-moderne, où le problème central devient la répartition des risques, transforme la perception et la construction du risque. Ce dernier n'est plus une menace extérieure, ou une fatalité, mais bien un élément constitutif de la société. Une telle approche renforce donc l'intérêt de concevoir l'évaluation d'un objet à risque comme les TI dans une optique sociétale. D'autant que, dans une telle société, ce n'est pas l'ampleur du risque qui change (il n'y a pas davantage de risques) mais sa «scientificisation » qui ne permet plus aux acteurs de mise en œuvre et aux groupes cibles de se décharger de leurs responsabilités en accusant la nature.

Face à la tension ainsi décrite, depuis une vingtaine d'années, notre société a développé des technologies, des procédures, des expertises et des agences administratives pour juguler les risques, singulièrement dans le secteur de la santé publique et sur des objets comme les TI. « Scientificisation » et « agencification » vont ainsi de pair et assument une triple fonction : réduire les incertitudes qui peuvent l'être, en mettant notamment l'accent sur l'activité de prévision ; réduire l'incohérence, en privilégiant l'efficacité par adaptation optimale et continue des moyens utilisés aux fins poursuivies ; réduire la diversité, en rendant compatibles les rationalités parfois contradictoires des nombreux acteurs ${ }^{22}$.

L'avis du CESE illustre cette tension : « Il faut certes prendre des risques en matière de recherche pour ne pas entraver le progrès et les découvertes, mais quand les applications des technologies clés génériques seront produites en grande série, le CESE souhaite qu'elles ne compromettent ni le bien-être de la population, ni 
la durabilité de l'environnement $\aleph^{23}$. Les risques ne sont pourtant pas définis dans le règlement (CE) n¹394/2007. Concernant plus spécifiquement les TI, le parlementaire belge du Bus de Warnaffe (cdH, démocrate-chrétien francophone), auteur d'une proposition de résolution relative aux TI, remarque que "l'émergence des médicaments de TI confronte les autorités nationales à des défis spécifiques $»^{24}$. Et c'est en ces termes que les acteurs de mise en œuvre et les groupes cibles construisent les risques liés aux TI. Des solutions elles-mêmes innovantes, inspirées des régulations antérieures, sont-elles alors mises en place pour réguler des biotechnologies innovantes?

Depuis les années 1990, une solution adoptée par les décideurs publics pour faire face à ce type de risques est l'installation d'agences administratives. Dans un contexte de gouvernance et de recomposition de l'action publique par des jeux d'échelles, l'agencification répond à une logique de décentrement. La régulation publique tend vers un régulateur indépendant et une expertise scientifique et technique poussée. Les TI n'échappent pas à ce qui semble devenu une règle.

Une illustration éclairante du rôle important dévolu à l'EMA (agence administrative à l'échelon européen) est fournie par le règlement (CE) $n^{\circ} 1394 / 2007$ (articles 4 et 5) : pour les essais cliniques et les bonnes pratiques de fabrication, c'est après avoir consulté l'EMA que la Commission européenne formule des lignes directrices. À l'échelon fédéral en Belgique, est clairement reconnue l'expertise du dispositif agentiel que constituent l'EMA, son comité d'experts (Le CAT) et l'Agence fédérale pour les médicaments et les produits de la santé (AFMPS), ainsi que le rôle qu'ils doivent jouer pour gagner la confiance des bénéficiaires finaux et des groupes cibles, dans un quasi lien de cause à effet : "L'évaluation des médicaments de thérapie innovante demande une expertise très spécifique, qui va au-delà du domaine pharmaceutique traditionnel (...). C'est pour cette raison que l'Agence européenne des médicaments a établi un nouveau Comité : le Comité pour les thérapies innovantes (...). Ce Comité a pour principale vocation d'évaluer la qualité, la sécurité et l'efficacité des médicaments de thérapie innovante, dans le cadre de la procédure européenne centralisée de mise sur le marché de médicaments. Il devrait dès lors disposer de la meilleure expertise possible, assurer un niveau élevé d'évaluation scientifique de ces médicaments afin de conserver la confiance des patients et des professions médicales et de faciliter l'accès de ces technologies novatrices au marché communautaire. L'Agence fédérale des médicaments et des produits de santé (AFMPS) est représentée dans ce Comité $\rrbracket^{25}$.

En matière de TI, la gestion et l'évaluation du risque sont donc maîtrisées par une structure homologue entre l'échelon européen et l'échelon fédéral (pour la Belgique), composée de régulateurs indépendants et de comités d'experts spécialisés. Au sein de l'EMA, le CHMP et le CAT sont en charge de la procédure centralisée d'AMM. L'AFMPS assume ce qui concerne les essais cliniques et les produits de thérapie innovante préparés de façon ponctuelle, à l'intention d'un malade déterminé, selon des normes de qualité spécifiques, sous la responsabilité professionnelle exclusive d'un médecin ${ }^{26}$. 
L'AFMPS reçoit également une mission de contrôle qui constitue une autre acception d'une évaluation limitée des TI. En novembre 2011, dans une question parlementaire adressée par Marleen Temmerman (sp.a, socialiste néerlandophone) à la ministre de la Santé publique, la fonction de contrôle par l'AFMPS est mise sur la sellette : «Quelle est la politique d'inspection de l'AFMPS en matière de thérapies innovantes? Entre-temps, combien de dossiers de demande d'essais cliniques ont-ils été traités ou approuvés par l'AFMPS? L'AFMPS doit-elle vérifier les bonnes pratiques de fabrication avant le début de la recherche? Comment s'opéreront les inspections de l'AFMPS? Élabore-t-on un modèle d'inspection adapté aux médicaments de thérapie innovante en première phase de développement, centrée sur la sécurité et la faisabilité ? $\aleph^{27}$. De telles questions, adressées notamment dans l'arène parlementaire, illustrent que le dispositif d'évaluation, entendue ici comme contrôle du risque, est un processus en évolution.

Dans une optique de précaution (théorisée comme une tension face à un risque dont les acteurs de mise en œuvre et les groupes cibles acceptent qu'il soit constitutif de notre société), ce processus passe par un système de pharmacovigilance européen renforcé. Actuellement en construction, il implique la mise en place d'un comité d'experts supplémentaire : le Pharmacovigilance Risk Assessment Committee (PRAC). L'EMA prévoit une consultation du public, en 2013, quant à l'élaboration des lignes directrices relatives au système de pharmacovigilance (GVP), par exemple sur le module 12 : «Continuous pharmacovigilance, ongoing benefit-risk evaluation, regulatory action and planning of public communication $»$. Globalement, c'est une conception techniciste du risque et de l'expertise qui prévaudrait encore, notamment avec une orientation comme celle du module 16 : «Risk-minimisation measures: selection of tools and effectiveness indicators 》 qui va dans le sens d'une étude d'impacts telle qu'abordée dans la première section de notre contribution.

Décrire l'existence des agences et des comités d'expertise pour attester de la structure décentrée et spécialisée prenant en charge la régulation des TI ne suffit pas à montrer la logique dominante qui prévaut pour leur régulation publique. Si nous avons montré jusqu'ici que le dispositif mis en place participe d'une conception relativement restrictive de l'évaluation centrée sur le rôle des agences administratives, il est important de mettre en avant les interactions entre, d'une part, les agences et les comités d'experts et, d'autre part, les acteurs de mise en œuvre, groupes cibles et bénéficiaires finaux.

Les acteurs de mise en œuvre mettent en place les agences et comités d'experts, notamment par les actes réglementaires qu'ils adoptent. Les groupes cibles (entendus comme producteurs de TI) sont des interlocuteurs privilégiés des agences. Le bilan des réunions informelles organisées par l'AFMPS entre le $1^{\text {er }}$ juillet 2010 et le 31 décembre 2010, dans le cadre de la Présidence belge de l'Union européenne, mentionne que « il peut être utile que des firmes soient invitées dès le début de la phase de développement pour présenter leur approche $»^{28}$. Récemment, l'EMA (avec l'Agence européenne pour la sécurité alimentaire, EFSA, et l'Agence européenne 
pour l'environnement, EEA) a pourtant essuyé les reproches du Parlement européen qui avait voté, en sa séance plénière du jeudi 10 mai 2012, le report de son budget suite à ce qu'il estimait être une gestion contestable des conflits d'intérêts entre l'agence et les groupes cibles ${ }^{29}$. Les interactions entre agences, experts et groupes cibles restent donc problématiques, sans qu'il soit possible de déterminer clairement à ce stade, pour les TI, si des leçons ont été tirées des difficultés dénoncées dans le cadre de ces interactions. Toutefois, dans le cas des TI, un élément intéressant, et sans doute nouveau concernant ces interactions, est le rôle potentiellement assumé par les bénéficiaires finaux. Comme le montre Delgalarrondo, les patients jouent sur une association plutôt que sur une opposition avec les groupes cibles et les agences ${ }^{30}$.

\section{Participation du public ou intervention de patients spécialisés ?}

La régulation publique des TI inaugureraitelle l'articulation plus poussée d'une logique représentative (marquée par le leadership des agences et des experts) avec une dynamique participative (par laquelle les bénéficiaires finaux seraient amenés à influencer davantage la régulation publique) ? Comme le relève la littérature scientifique sur les politiques du risque, une réponse positive à cette question ne va pas de soi : le public (il s'agirait, dans nos termes, des bénéficiaires incluant, non seulement les patients, mais les citoyens de manière plus large) fait peur. Borraz, entre autres, explique que la société du risque n'induirait pas un État plus participatif que représentatif (appelé de ses vœux par un théoricien de la démocratie comme Blondiaux), mais plutôt des acteurs de mise en œuvre activant des modalités quasi technocratiques de production et d'implémentation de règles faisant appel à la concertation, aux débats, aux controverses ${ }^{31}$. Les risques potentiels des TI seraient donc débattus comme l'ont été d'autres risques (téléphonie mobile, OGM, nucléaire, etc.).

Quelle peut être alors l'intervention des patients et citoyens sur ces risques complexes? L'avis du CESE illustre cette tension supplémentaire entre le fait de tenir compte du public et de limiter le frein qu'il peut constituer au développement des connaissances et de la régulation : « la Commission [européenne] s 'inquiète à juste titre du fait qu'en l'absence d'une information correcte, l'opinion publique peut être victime de désinformation et s'opposer, sans fondement raisonnable, à l'introduction de produits ou de services basés sur des technologies clés génériques. Il convient en toute priorité d'intéresser les citoyens en général, et plus particulièrement les jeunes, aux applications extraordinaires de la science et de la technologie que nous côtoyons chaque jour $\rangle^{32}$.

Dans le cas des TI, une consultation du public est prévue à l'occasion de la mise au point des lignes directrices par l'EMA. En théorie, elle doit impliquer «toutes les parties intéressées » mais en pratique, elle ne permet pas un accès des profanes à la régulation publique. Ceci s'explique en grande partie par la complexité des enjeux qui ne sont pas accessibles au grand public, tout au plus aux patients. L'esprit du règlement (CE) $n^{\circ} 1394 / 2007$ est clair dans le «considérant » $\mathrm{n}^{\circ} 21$, les acteurs de mise en œuvre et l'industrie pharmaceutique (groupe-cible) sont explicitement cités comme acteur-ressource : «Il y a lieu de 
procéder à une consultation ouverte à toutes les parties intéressées, en particulier les autorités des États membres et l'industrie, afin de permettre la mise en commun des compétences, limitées dans ce domaine $»$.

L'article 21 du règlement (CE) n¹394/2007 prévoit que deux membres (avec deux suppléants) représentent les associations de patients au sein du CAT. Ce mécanisme s'inscrit dans une dynamique représentative davantage que dans une dynamique d'influence directe des patients (bénéficiaires finaux) sur la régulation publique des TI. Mais la démocratie, particulièrement dans des domaines pointus comme les TI, d'une complexité scientifique, médicale et technique avérée, suppose-t-elle la participation directe du public à la régulation publique, à son évaluation, à l'expertise de l'objet ? Cette nouvelle tension a été exprimée dans les termes d'un dilemme démocratique par Dahl ${ }^{33}$.

Dahl met en exergue l'ambivalence entre « the ability of the citizens to exercise democratic control over the decisions of the polity versus the capacity of the system to respond satisfactorily to the collective preferences of its citizens $\|^{34}$. Dahl abordait la problématique de la capacité d'action des citoyens selon que les décisions sont prises à un niveau plus ou moins éloigné d'eux : du local à l'européen, voire au supranational. Le jeu d'échelles qui affecte la régulation publique d'objets comme les TI coïncide avec le propos de Dahl. De plus, Dahl pointe l'intervention des citoyens (dont ici les patients) en termes de dilemme entre participation et efficacité (un des critères d'évaluation) d'une régulation publique (dilemme accru lorsque cette dernière porte sur des enjeux complexes comme les TI).

En Belgique, l'intervention des bénéficiaires finaux dans le secteur des TI s'est réalisée par un processus de judiciarisation. En effet, trois associations ont introduit un recours devant la Cour constitutionnelle. Les associations sans but lucratif (ASBL) « Jurivie », « Pro Vita » et « Jeunes pour la vie » ont demandé à ce que des dispositions de la loi belge du 19 décembre 2008 transposant la directive 2004/23/CE soient annulées. Les deux associations contestaient les définitions données aux notions d'embryons et de fœtus (l'objectif des associations étant de défendre le droit à la vie). D'après les associations, la loi affectait la dignité de la personne dès sa conception, ce qu'ont récusé le Conseil fédéral des ministres et la Cour constitutionnelle ${ }^{35}$.

Pour Rosanvallon, il est possible de voir dans un tel processus de judiciarisation une dimension importante de la dynamique démocratique contemporaine : la montée en puissance de bénéficiaires finaux adoptant une posture de juge par rapport aux acteurs de mise en œuvre. Dans un secteur à risque et controversé comme les TI, cette dimension revêt une importance particulière. Elle participe, comme la dénomme Rosanvallon, d'une " contre-démocratie qui n'est pas le contraire de la démocratie ; c'est plutôt la forme de démocratie qui contrarie l'autre, la démocratie des pouvoirs indirects disséminés dans le corps social, la démocratie de la défiance organisée face à la démocratie de la légitimité électorale $»^{36}$. Elle complète l'aperçu des modalités possibles d'intervention des bénéficiaires finaux dans le secteur. 


\section{Conclusion}

Notre contribution a montré que la régulation des TI dénote une relative hybridation. Celle-ci a été déclinée en trois points : (1) expertise ciblée sur des objets scientifiques et médicaux plus qu'évaluation globale d'une politique publique, mais prise en compte des traits de la société du risque dans laquelle s'applique la régulation ; (2) prépondérance de l'expertise pointue, mais ouverture disciplinaire notamment à la gestion des risques et à l'éthique ; (3) délégation aux agences administratives incluant des interactions privilégiées avec les groupes cibles, mais ouverture au public via des consultations.

Un premier constat tiré ici est que les acteurs de mise en œuvre anticipent ce que peut être la régulation publique des TI. Cette réflexion arrive plus tôt que n'était arrivée celle sur le tabac ou l'amiante, par exemple. Qui plus est, elle organise une information et/ou une consultation du public, et intègre la participation de bénéficiaires finaux (patients) disposant d'une expertise d'usage. Ces derniers peuvent aller jusqu'à s'approprier l'objet en enclenchant un processus de judiciarisation.

Un second constat est que les acteurs de mise en œuvre intègrent l'enjeu éthique des TI. Mais opérationnaliser l'articulation entre éthique, science et action publique dans le cadre d'une évaluation de politique publique reste encore un chantier. L'option reste réglementaire. Or (1) le droit possède une temporalité plus longue, une inertie propre à la longueur de l'adaptation des normes et (2) la science n'a pas, déjà, toutes les réponses aux questions posées par le caractère émergent des TI et qui pourraient être réglementées. En Belgique, les décideurs publics notent la nécessité, pour les cadres réglementaires qui viendraient en complément de la régulation actuelle, de ne pas être "rigides ou figés, mais plutôt souples et évolutifs, car les progrès scientifiques dans le domaine des biotechnologies évoluent très rapidement et requièrent des adaptations réglementaires régulières $»^{37}$.

Le troisième constat est que la littérature scientifique doit donc encore investiguer la triangulation entre (1) accepter la prise de risque qu'impliquent les développements scientifiques émergents, (2) produire une action publique qui équilibre innovation et précaution, avec (3) une éthique qui ne soit pas ultra-spécialisée sur l'objet scientifique dans ses dimensions éthiques et qui associe le public. Une appréhension sociétale des nouveaux risques permettrait de réfléchir ensemble : crainte du public, enjeux économiques des innovations, temporalités du décideur public. 
$\mathrm{N} \cdot \mathrm{O} \cdot \mathrm{T} \cdot \mathrm{E} \cdot \mathrm{S}$

1. Voir supra, Florence Taboulet, « Les médicaments de thérapie innovante : quelles spécificités en droit pharmaceutique?».

2. Avis du Comité économique et social européen (CESE) sur la communication de la commission au Parlement européen, au Conseil, au Comité économique et social européen et au Comité des régions, « Préparer notre avenir : développer une stratégie commune pour les technologies clés génériques dans l'UE », 2001/C 48/20, p. 114.

3. La littérature sur ces constats scientifiques abonde. Une synthèse parmi d'autres est fournie par Gilbert $C$., Lascoumes P., «Les politiques des risques en Europe », Revue Internationale de Politique Comparée, vol. 10, $\mathrm{n}^{\circ}$ 2, 2003, pp. 151-160.

4. Parmi les ouvrages les plus pertinents aujourd'hui, retenons Rossi P. et al., Evaluation. A systematic approach, Thousand Oaks, Sage Publications, 2004.

5. Règlement (CE) $n^{\circ} 1394 / 2007$ du Parlement européen et du Conseil du 13 novembre 2007 concernant les médicaments de thérapie innovante et modifiant la directive 2001/83/CE ainsi que le règlement (CE) n726/2004; Directive 2004/23/CE du Parlement européen et du Conseil du 31 mars 2004 relative à l'établissement des normes de qualité et de sécurité pour le don, l'obtention, le contrôle, la transformation, la conservation, le stockage, et la distribution des tissus et cellules humaines ; Loi du 19 décembre 2008 relative à l'obtention et à l'utilisation de matériel corporel humain destiné à des applications médicales humaines ou à des fins de recherche scientifique, Moniteur belge 30 décembre 2008.

6. De Visscher C., Varone F., Évaluer les politiques publiques. Regards croisés sur la Belgique, Louvainla-Neuve, Academia-Bruylant, 2001, p. 7.
7. Selon Kingdon, une fenêtre d'opportunité est l'occasion fournie aux porteurs d'une cause d'avancer leurs propositions et d'attirer l'attention sur des problèmes spécifiques. Kingdon J., Agendas Alternatives and Public Policies, Boston, Little Brown, 1984.

8. Rossi, op. cit., p. 235

9. Voir supra, Alessandro Blassimme, « Translating stem cells to the clinic: scientific societies and the making of regenerative medicine $»$.

10. Knoepfel P. et al., Analyse et pilotage des politiques publiques, Zurich: Rüegger, 2001, pp. 253-287.

11. Voir notamment Comité consultatif de Bioéthique de Belgique (CCB), avis n52 du 12 mars 2012 concernant les aspects éthiques de certaines dispositions des règlementations européenne et belge en matière de tissus et cellules humains utilisés dans le cadre de la médecine reproductive, version définitive.

12. CCB, ibid., p. 3.

13. Agence nationale de sécurité du médicament et des produits de santé, Médicaments de thérapie innovante, médicaments de thérapie innovante préparés ponctuellement et préparations : synthèse $d u$ cadre réglementaire applicable pour la fabrication, le développement et la mise sur le marché de ces produits, version du 30 mars 2012 mise à jour le 5 juin 2012, p. 9 . 14. CCB, avis $n^{\circ} 45$ du 19 janvier 2009 relatif aux banques de matériels corporels humains destinés à la recherche, version définitive, pp. 3-4.

15. Gusfield J., La Culture des problèmes publics. L'alcool au volant : la production d'un ordre symbolique, Paris, Économica, «Études sociologiques », 2009 [1981].

16. Jobert B., Muller P., L'État en action. Politiques publiques et corporatisme, Paris, Presses universitaires de France, 1987.

17. Gardella Édouard, Boire ou conduire, La vie des idées.fr : http://www.laviedesidees.fr/IMG/ 
pdf/20090910_gusfield.pdf (date de consultation : 4 décembre 2012).

18. Short, J.F., « The social fabric at risk: toward the social transformation of risk analysis », American Sociological Association, ${ }^{\circ}$ 49, 1984, p. 711.

19. Debia M, Zayed J., «Les enjeux relatifs à la perception et à la communication dans le cadre de la gestion des risques sur la santé publique ", Vertigo, vol. 4, n 1, 2003, p. 2.

20. Peretti-Watel P., Moatti J.-P., Le principe de prévention, Paris, Éditions du Seuil et La République des Idées, 2009, p. 11.

21. Van Griethuysen $\mathrm{P}$, « Pour une approche évolutive de la précaution », Revue Européenne de Sciences Sociales, vol. 42, n ${ }^{\circ}$ 30, 2004, p. 156.

22. Brunet S., Schiffino N., «Évaluation et gestion des risques ", in Brunet S. et al., Articuler risques, planification d'urgence et gestion de crise, Bruxelles, De Boeck.

23. Avis du CESE, op. cit., p. 113.

24. Du Bus de Warnaffe C., «Proposition de résolution relative aux défis posés par l'émergence des médicaments de thérapie avancée ", Sénat de Belgique, session de 2010-2011, 9 septembre 2011, 5-1208/1, p. 5.

25. Du Bus de Warnaffe, op. cit., p. 2.

26. Article 28 du règlement (CE) n 1394/2007.

27. Temmerman M., « La préparation de thérapies innovantes à partir de cellules et de tissus d'origine humaine en hôpital », Question écrite $n^{\circ}$ 5-3682 à la vice-première ministre et ministre des Affaires sociales et de la Santé publique, chargée de l'Intégration sociale, 16 novembre 2011.

28. http://www.fagg-afmps.be/fr/binaries/tableau $\% 20$

bilan\%20fr\%2014042011_tcm291-122024.pdf_(date de consultation : 4 décembre 2012).

29. Le Monde du 10 mai 2012.

30. Delgalarrondo S., « Quelle place pour les asso- ciations de malades dans le processus d'innovation médicamenteuse ? ", Revue française des affaires sociales, 3, n 3-4, 2007, pp. 171-191.

31. Borraz O., Les politiques du risque, Paris, Science Po Les Presses, 2008 ; Blondiaux Loïc, Le nouvel esprit de la démocratie, Paris, Le Seuil, 2009.

32. Avis du CESE, op. cit., p. 113.

33. Dahl R., «A Democratic Dilemma: System Effectiveness versus Citizen Participation », Political Science Quarterly, vol. 109, n¹, 1994, pp. 23-34.

34. Dahl, ibid., p. 28.

35. Cour constitutionnelle, $a r r e ̂ t ~ n^{\circ} 68 / 2010$ du 10 juin 2010 et $\operatorname{arrêt} n^{\circ} 146 / 2011$ du 5 octobre 2011.

36. Rosanvallon P., La contre-démocratie, Paris, Seuil, p. 16.

37. Du Bus de Warnaffe, op. cit., p. 3. 


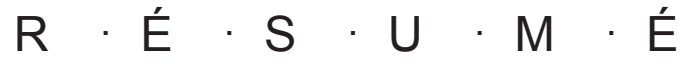

Notre contribution s'articule autour de trois notions clés : évaluation, risque et démocratie. Les thérapies innovantes questionnent la façon dont nos démocraties contemporaines évaluent le risque sanitaire à travers le prisme des leçons potentiellement acquises depuis trois décennies. Les analyses en science politique abondent sur la régulation publique des biotechnologies et sur leurs liens avec l'appréhension du risque et la mise en place de dispositifs décisionnels experts ou participatifs. Les thérapies innovantes défient pourtant aujourd'hui nos schémas de compréhension et d'explication de l'évaluation des risques sanitaires dans le secteur des biotechnologies.

L'article pose l'hypothèse que l'évaluation de politiques publiques dans ce secteur reste partiellement à inventer pour inclure une dimension sociétale. Pour la tester, il développe trois axes problématiques. L'article discute la notion et la pratique d'évaluation dans ce secteur. Classiquement, l'évaluation d'une politique publique intervient dans sa phase de terminaison (Jones) et vise à déterminer l'efficacité, l'efficience et la pertinence du programme d'action publique mis en œuvre. Il s'agit donc d'un diagnostic ex-post posé une fois que la politique publique ressort ses effets. En matière de TI, une évaluation ex-ante au cas par cas, sur l'objet à réguler davantage que sur une action publique d'ensemble, semble s'imposer. L'approche du Technology Assessment, renforçant le rôle des agences administratives (Benamouzig et Borraz), n'est pas étrangère à cette conception. Elle fait explicitement référence au risque et à la façon dont ce dernier est appréhendé. Les régulateurs sont les acteurs prédominants de l'évaluation, en association avec les parties prenantes. Dans cette perspective, il faut examiner quelle place est attribuée aux patients et plus largement aux citoyens dans l'évaluation des TI. C'est la légitimité primaire et secondaire (Scharpf), en termes d'inputs par la représentativité des décideurs et d'outputs par les décisions de régulation produites.

\section{Abstract \\ Advanced therapies through public policies and risk assessment}

Our contribution focuses on three key notions: assessment, risk and democracy. Advanced therapies are questioning the way our contemporary democracies are assessing health risk through the prism of the lessons potentially learned for three decades. Many analyses in political science are related to the public regulation of biotechnology and their relationship with the perception of the risk and the establishment of experts or participatory decision-making processes. Nevertheless advanced therapies nowadays challenge our patterns of understanding and explanation of the assessment of health risks in the biotechnology sector. The article makes the hypothesis that the evaluation of public policies in this area has still to be partially invented to include a social dimension. This hypothesis will be tested through three main issues.

The article discusses the concept and practice of evaluation in this area. Conventionally, a public policy is assessed in its termination phase (Jones) and aims to determine the effectiveness, efficiency and relevance of the public agenda implemented. It is therefore a diagnostic ex-post established once the public policy has produced effects. In the field of advanced therapies, an ex-ante evaluation on a case by case basis, more on the object to be regulated rather than on the public action at a whole, seems to be necessary. The approach of Technology Assessment, strengthening the role of 
administrative agencies (Benamouzig and Borraz), is not ignoring this concept. It makes explicit reference to risk and how it is understood. Regulators are the dominant players of the evaluation, together with stakeholders. In this perspective, we must examine what role is given to patients and to the wider public in the evaluation of advanced therapies. This is the primary and secondary legitimacy (Scharpf) in terms of the representativeness of the inputs by decisions makers and outputs by regulatory decisions produced. 
